\title{
INFLUENCE OF THE EU INSTRUMENTS ON HIGHER EDUCATION AND SCIENCE IN BULGARIA
}

\author{
Albena Vutsova ${ }^{1}$
}

\begin{abstract}
The European instruments have started acting adequately in Bulgaria since 1991, when the country was accepted as a member of a number of initiatives and pre-accession instruments were introduced. At a later stage commenced the action of the principal European instruments: the framework programs (1998) and structural funds (2003). They intervened the research and education area as real tools in the late 1990 and are acting up to now, being either options from new programing period of Structural funds functioning or as initiatives of new generation Framework programs (Horizon 2020 and Erasmus+, etc.)

This article considers the positive effects of these instruments in three directions consecutively: Financially, as a source of additional resources; Science Metrics-wise, as a volume of scientific production, created with their financial support, and evaluation of this production by the world scientific community; Other benefits.

The effect of realizations of this instruments has been explored and achieved results have been analyzed as a fundament for further improvement of their effective absorption and bettering the system of research and education.
\end{abstract}

JEL Classification Numbers: D83, I23; DOI: http://dx.doi.org/10.12955/cbup.v5.1043

UDC Classification: 00

Keywords: education, research, knowledge, EU, instruments, Bulgaria

\section{The European instruments - pre-accession instruments}

The European instruments have started acting in Bulgaria since 1991, when the country was accepted as a member of a number of initiatives and pre-accession instruments were introduced. At a later stage commenced the action of the principal European instruments: the framework programs (1998) and structural funds (2003).

PHARE Programme was the first program, applied to Candidate-States for EU membership, in order to prepare their institutions for full membership in the Community. PHARE was directed to almost all sectors of the economy. Later supplemental pre-accession instruments were introduced - the programs ISPA (Instrument for Structural Policies for Pre-accession) and SAPARD (Special Accession Programme for Agricultural and Rural Development). PHARE program began to act in Bulgaria in 1991 with total financial support in the first stage (1993-2001) - Euro 11.0 million. During 2004-2006 Euro 14.0 million were provided to units of the system for the Education activity. As indirect support, according Borras (2012) for the scientific-innovative activities can be considered the development of the National Innovation Strategy, described by Freeman (1995), and the National Innovation Fund to support the innovation activities of SMEs, in a partnership with scientific organisations and universities presented by Bendis and Byler (2009). Total financial value of this scheme was Euro 1,179 million. For the period 2005-2007 the funds, provided to higher education system and Bulgarian Academy of Sciences (BAS) under the PHARE program, were insignificant (1\%) relative to the means, provided by the state budget.

Bulgaria joined the TEMPUS program officially in 1991. From the beginning of the action of this program (1990-2006) it has provided the participating countries a total of Euro 300 million. The program had a positive effect with respect to the development of project culture and sustainable partnerships. During the active participation of the country, 1993-2001 (second program period), the universities received Euro 68,930 million. If we compare the data about the support received by the other countries, which were also in the pre-accession stage, Bulgaria ranks a good fourth position after Poland, Romania, and Hungary.

The program Erasmus emphasizes on the university structures and supports the mobility of lecturers and students. Since the beginning of its action in 1987 more than 3 millions of people have used the opportunities for academic exchange. It is a principal instrument for the realization of the educational priorities of the Community. Bulgaria joined the program in 1999. During the first academic year of activity of the ERASMUS program in Bulgaria (1999-2000) only 10 Bulgarian high schools received

\footnotetext{
${ }^{1}$ Prof., Faculty of Economics and Business Administration, Sofia University “St. Kliment Ohridski”, avutsova@yahoo.com
} 
financing for mobility, but partnerships were developed with 42 European universities. The development of the program leads to systematic increase of the funds provided for mobility.

\section{European instruments used by NMS}

The basic instruments of the harmonization policy are the structural funds - the European Regional Development Fund (ERDF) and the European Social Fund (ESF). The means provided for science and education during 2007-2013 by the structural funds amount to Euro 557,740 million, where these financial resources also include the funds provided for secondary education. The principal measures, supporting higher education and science, are initiated in three of the operational programs "Development of the Competitiveness of the Bulgarian Economy" Operational program (DCBEOP), "Development of the Human Resources" (DHROP) Operational program, and the "Regional Development" Operational program (RDOP). 120.747 million Euros of the resources for the priority axis 1 (development of the economy, based on knowledge and innovation activities) of DCBEOP is directed to the development of scientific organizations, research and infrastructure, bound to the business and economic development of the country. 8,446 million Euros was provided to support the research infrastructure of scientific organizations - only BAS institutes. Euro 42.825 million or $25.19 \%$ of the total Euro 105.386 million support were allocated specifically for innovative scientific activities.

HRDOP has provided directly to its beneficiaries a total of 32.876 million Euros. In the program period 2007-2013 various procedures have been carried out.

RDOP is focused on improving the infrastructure of universities. The funds provided via RDOP (Euro 18.402 million) went to 15 universities only and the actions of the OP have completed entirely. Since these funds were oriented to subjects of higher education, it is considered correctly this amount to be accounted for financial income and indirect contribution regarding the improvement of the educational and research process.

The framework programs for research and innovation are primary instrument of ERA (European Research Area) as per COM (2012) and COMPET 174(2008, May 16). They guarantee the execution of the Community policy in this area. Bulgaria started its participation in the limited activities of the third framework program. In the last program period of the framework programs Bulgaria has received Euro 11.4 per capita. For comparison, the Czech Republic has received Euro 19 per capita, Hungary Euro 22.2, Romania - Euro 5.6. The average value for the new Member States is Euro 13.5 according to Knee et al. (2011).

The ratio between the amount paid by the country as a member of the EU, and the income from the framework programme for one year shows, that through this instrument the country gets back $4.3 \%$ of the "membership fee" Eurada (2014), which is not high, but combined with the resources from other similar instruments, creates conditions for a sustainable synergy.

INTERREG program was comparatively new to Bulgaria. Its beginning was in 2002 as a regional program, operating with financial support from the EU. The participation of Bulgaria in the first period was limited to a number of projects with no leadership. The overall contribution is Euro 10178 million.

First European program for science and technology COST supports primarily horizontal and coordination activities. During the last several years the financial support, received by Bulgaria for the above mentioned activities, has grown steadily.

\section{EU instruments - Influence and benefits}

Since the GDP of the country is a fundamental characteristic of the national economy, and the latter in its turn is a reliable financial resource for science and education, a simplified mathematical model for its forecasted development in time has been elaborated.

The expenditure of the government and businesses for research as a percentage of the GDP are illustrated in Figure 1. 


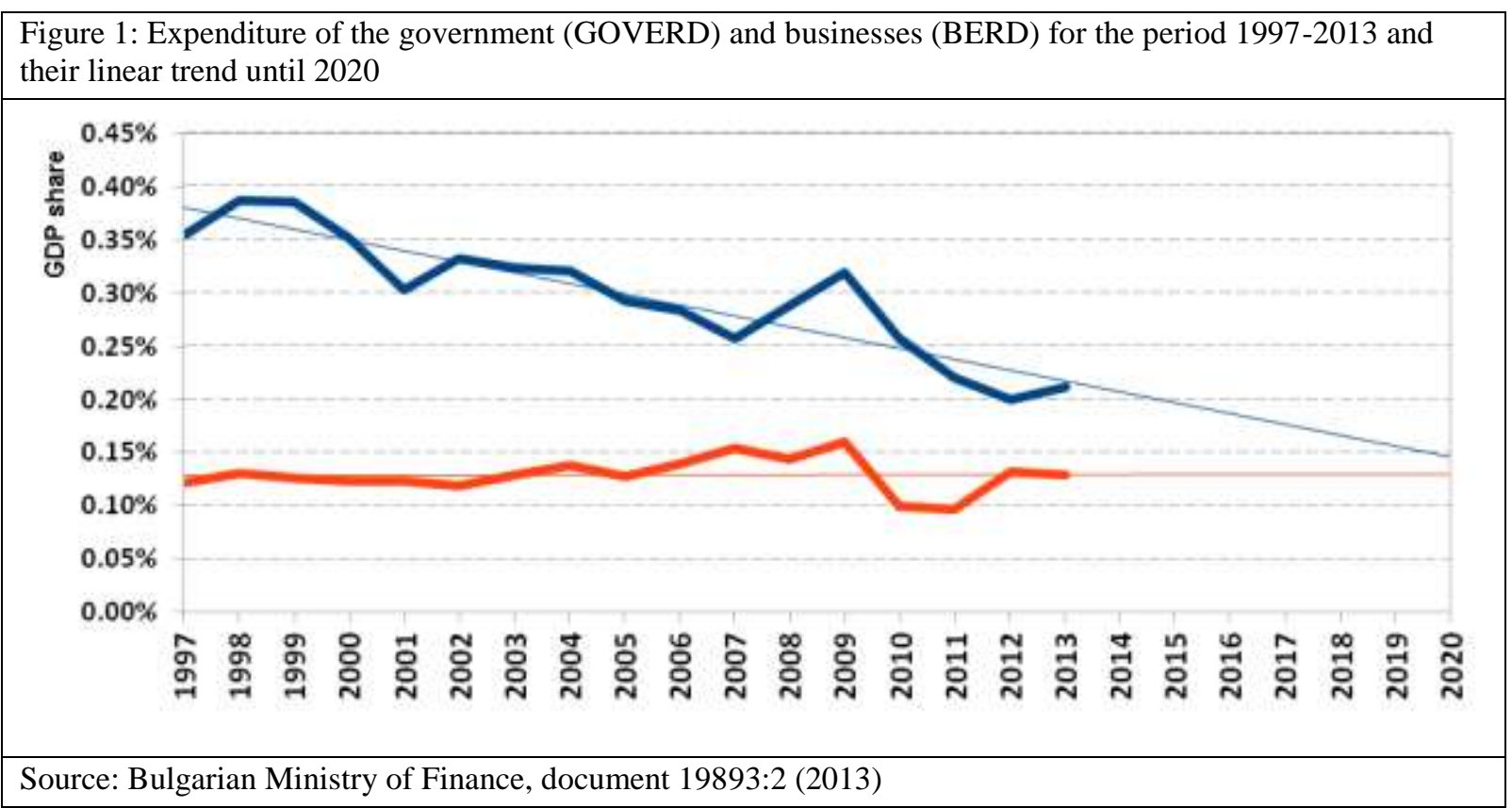

The reduction of government expenditure and the sustainable trend with the expenditure of businesses are obvious. The trend of the reduction of the government expenditure must not necessarily fall below $0.15 \%$ of GDP, as shown in the graphic, but the sustainable trend of reduction will obviously continue, and without any specific efforts the government expenditure will remain at the current levels. It means that the importance of EU instruments as steady resources are growing.

\section{Scientometric data and effect}

PHARE and TEMPUS, created the first effective partnerships with European scientific institutions for carrying out joint research, obtaining and publishing of a considerable number of high value results. Bulgaria has tried to use both instruments actively. Greece and Ireland exhibit similar behavior, but they do not combine the two instruments. During the same period the Czech Republic uses the instruments significantly less, and Spain does not use them for this purpose at all probably because the national financing of science in these countries is much better.

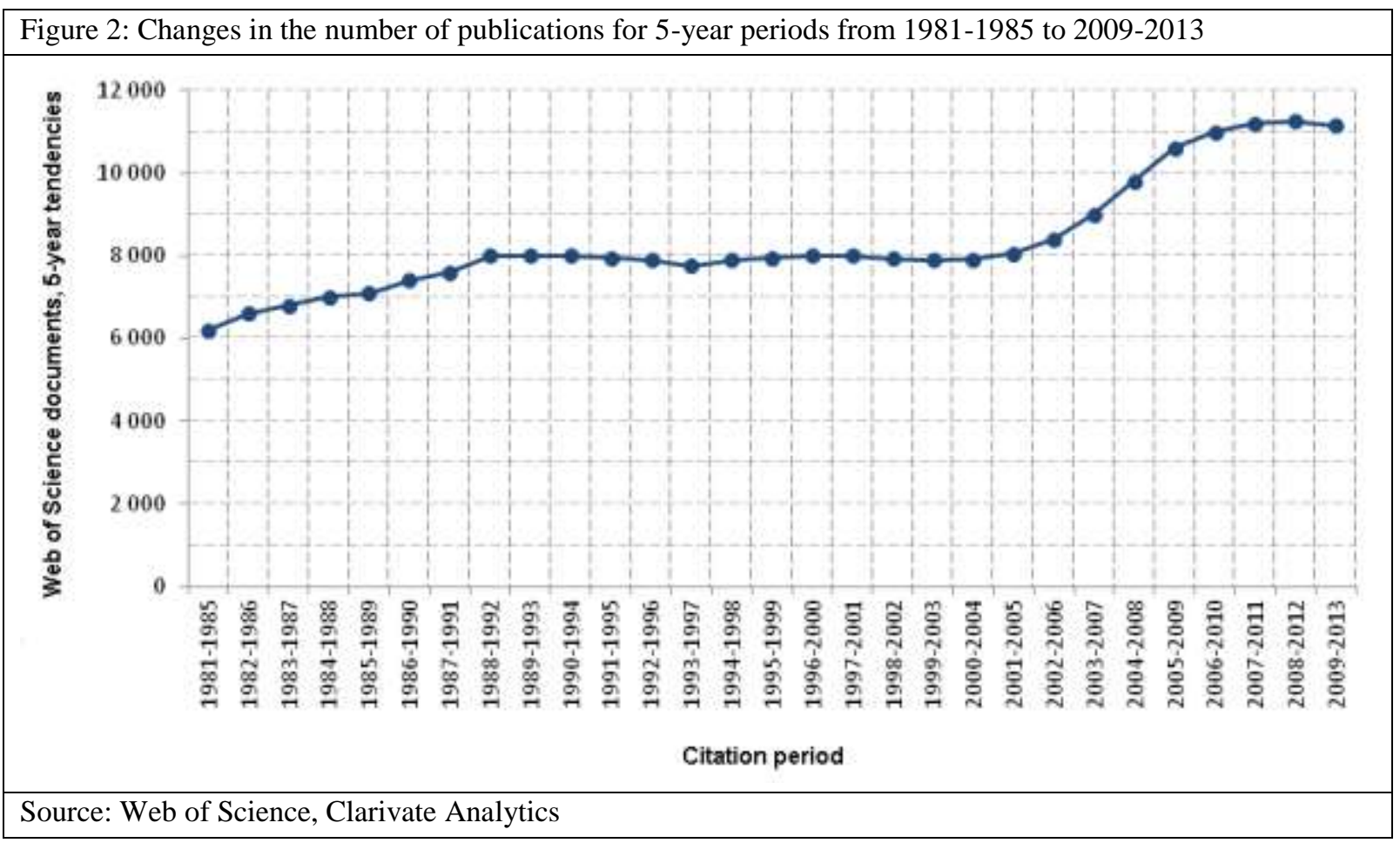


Obtaining the right for full access to the Framework programs and the COST program the Bulgarian scientific community became more active, The evaluation in this work covers FPs 5, 6, and 7, since these are the FPs, in which Bulgaria has full access to all types of activities. During each program the scientific community gathered expertise and by also using the opportunities of the other European instruments achieved a synergic effect (such an example was the parallel participation in the COST program, financed via FP). The bibliographic data of Bulgaria are compared to the same reference countries, as above.

The results for FP 5 show clearly, that for Bulgaria all parameters have low values because at this stage the experience was very small. FP 6 observed some positive changes. By a total number of scientific publications Bulgaria prevails over the Czech Republic and approaches the achievement of Austria, i.e., the publication activity is at an acceptable level, especially bearing in mind the above observations. According to this graphic, Bulgaria has improved part of its metrics. With respect to the percentile however (the values are reversed, as pointed out in the note about the network graphics), Bulgaria's characteristic is still the lowest.

\section{FP 7 influence upon scientific productivity}

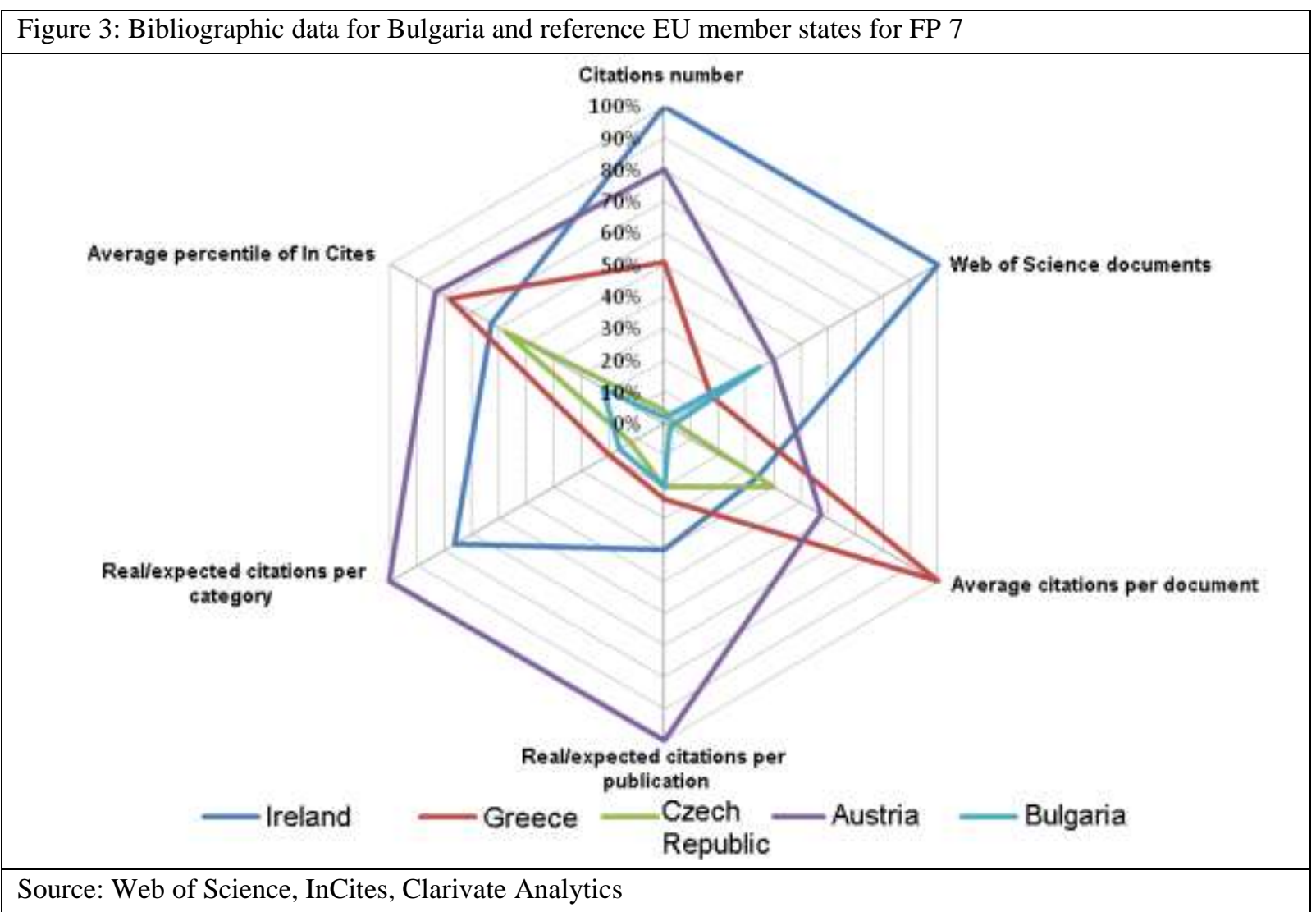

With FP 7 the picture changed with respect to some of the characteristics, reflecting the gained experience and created more sustainable partnerships with leading European research centers. The noticeable improvement of the scientometric data was due, to the provision of constant access to Elsevier's and Clarivate Analytics'(ex Thomson Reuters) science metric databases. Following long years of practically discontinued conventional library access to scientific literature and information, another, new culture of their intensive use was created, and the Bulgarian scientists joined actively in this efficient avenue of knowledge exchange.

The use of the Internet platforms for scientific information provides an opportunity to trace also any individual publication activity and to produce a ranking of the researchers. An analysis of the bibliometric data shows, that the participants in European projects are among the best and most active researchers in their universities and research centers. 


\section{Bilateral cooperation - influence upon scientific productivity}

With respect to the Hirsch-index (h), according Toshev 2011, of the number of publications, which according to quote a few authors is a sign of the development of the scientific sector for the period since the beginning of operation of the European instruments to this day, Bulgaria is ranked 21-st in EU-27 with an h index of 154 (for the sake of comparison: Great Britain has 934, and the Netherlands - 636; data SCOPUS, Science in European Union 2013). Lower ranking countries include Romania, Estonia, and Malta.

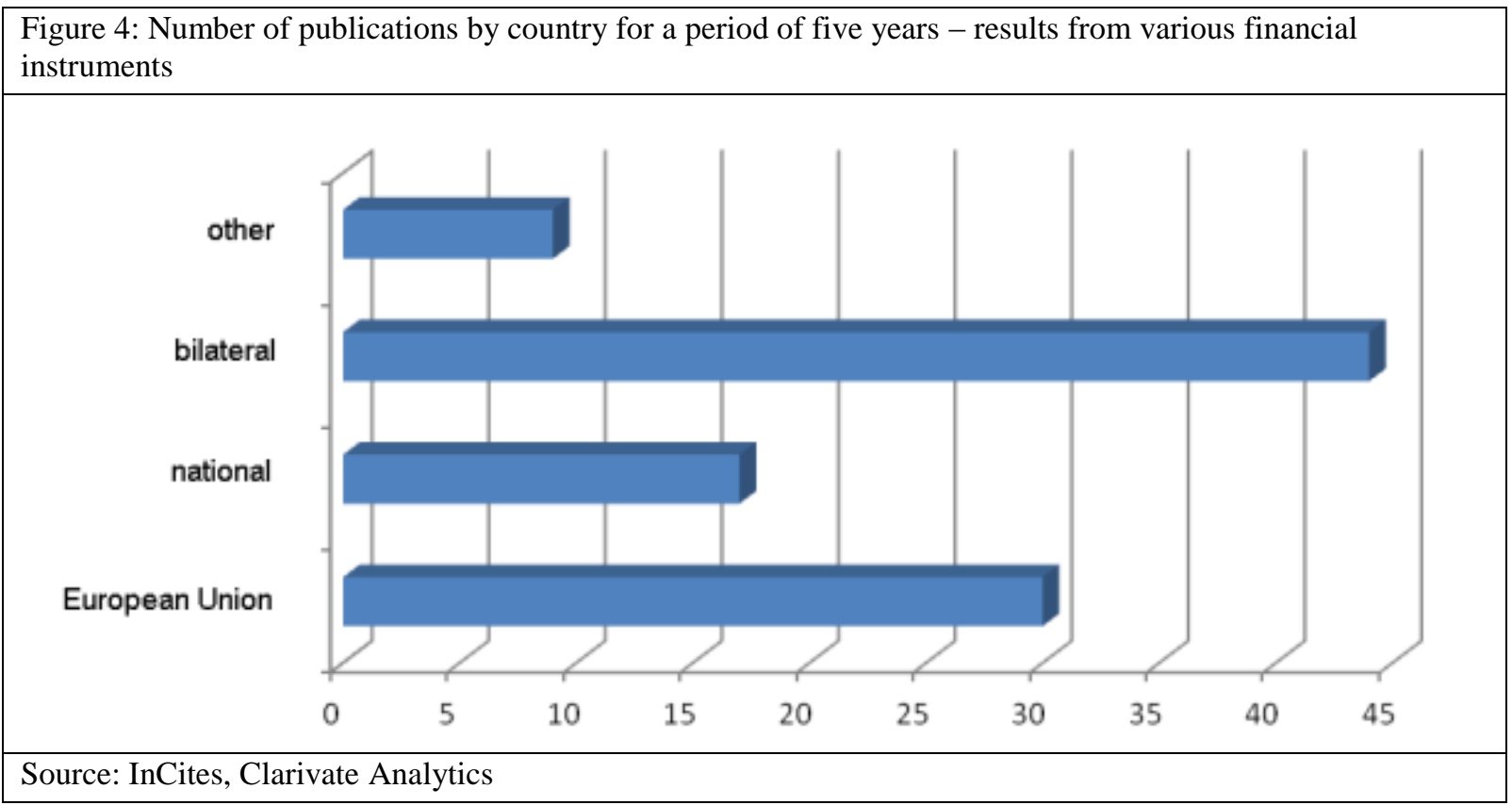

In the context of Europe 2020 the target is the financing of science to reach $3 \%$ of GDP, and, moreover, the prevailing contribution (2/3) is to come from businesses. Exactly businesses finance their own research, which quite often do not lead to publications, but due to the growing competitiveness of the environment - mostly to patents. E.g., only two corporations in Germany (SIEMENS and DAIMLER) invest in proprietary research 5 times more funds, than the financed primarily by the government research organization Max Planck, doing primarily basic research.

\section{Benefits from EU instruments - general, as per SEC (2010) and EUCO 13/10 (2010, June 17)}

Under the action of the pre-accession instruments in Bulgaria were created conditions for:

- development and consolidation of the administrative capacity of the government bodies, and educational and scientific institutions, so that the administration to be capable of operating in the EU environment;

- harmonization of the legislation in the higher education and science system, in order to shorten the transition periods according to Simeonova-Ganeva and Ganev (2013);

- progress in the development of institutions, to allow the creation and development of structures, strategies, human and managerial resources for strengthening the economic, social, and legislative capacity studied by Horvat (2013);

- introduction and use of specific instruments, like, e.g., the Twinning initiative, oriented to direct acquiring of good practices and experience in specific directions from similar institutions in the EU. This instrument provides technical and expert support, resulting in increased capacity of the human resources described by Ahrens (2006);

- introduction to specific instruments, complementing the primary ones, like the "Trans-border collaboration" programs, supporting the active development of capacity of the institutions, preparing them for participation in the European programs.

TEMPUS and ERASMUS programs influence the partial restructuring of the higher education system according to Inayatullan and Gidley (2000) as a whole - changes of the general network of HSs, as well as changes in the internal structure of some HSs. Supplemental contributions according to 
Jongsma (2002) are : creation of strategic documents for the development of the higher education system based on partnerships; long-term effect in the universities as partners in the knowledge triangle; long-term effect on the internal-institutional structure: chairs, departments, faculties; introduction to internal and national systems for quality assurance.

Thus conditions are created for: higher quality and better efficiency of the educational process, which, in turn, influences the raising of a generation with new knowledge and skills; introduction of the European credit system; introduction of innovative and/or non-traditional methods of teaching and training described by Keegan (1990), which creates opportunities for the development of key competencies in various groups trainers and trainees; consolidation of the social harmonization at various levels;

The accumulation of knowledge as a result of the operation of various types of instruments for science and innovation can be considered also as a specific "warehouse/reservoir" for new knowledge or a "depositary", which according to the World Bank (2003) is a set of the following specific activities: creation of knowledge; application of knowledge; sharing of knowledge with partners and peers by new products and services.

With the creation of innovation products, representing an integrated result of the creation of new or improved processes, products, and services based on new or improved know-how, a positive trend is observed. This is an important result of the action of European instruments, since the innovation product is one of the criteria for a functioning innovation system. For the last 10 years a growth of almost $20 \%$ is observed with respect to the registration of trademarks.

\section{References}

Borras, S. (2012, February 17). "EU politics in research and innovation from Government to governance," 2003 Commission recommendation 5-14.

COM (2012). Communication from the Commission to the European Parliament, the Council, the European economic and social committee and the Committee of the regions: A Reinforced European Research Area Partnership for Excellence and Growth, 392 final.

COMPET174 (2008, May 16). Council Conclusions on the launch of the "Ljubljana Process" - towards full realisation of ERA, 9029/1/08 RECH 161168

Toshev, B. (2011). Scientific competence - a personal and institutional evaluation, Criticism and Humanism, 36 (1), 119134.

Eurada (2014). DG Research \& DG Budget reproduced in Eurada.

Arnold, E., Mahieu, B., Stroyan, J., Campbell, D., Carlberg, M., Giaracca, F., Horvath, A., Jávorka, Z., Knee, P., Meijer, I., Sidiqi, S., Wagner, C. (2011). Understanding the Long Term Impact of the Framework Programme, European Policy Evaluation Consortium.

SEC (2010). European Commission Lisbon Strategy evaluation document. Commission Staff Working Document.

EUCO 13/10 (2010, June 17). European Council Conclusions.

Freeman, C. (1995). The National System of Innovation in Historical Perspective, Cambridge Journal of Economics, 19, 524.

Inayatullan, S. and Gidley, J. (2000). Forces facing University future In the University in transformation.

The World Bank (2003). Innovative East Asia: The Future of Growth, The International Bank for Reconstruction and Development 231.

Ahrens, J. (2006). Goverance in process of economic transformation, 2.

Desmond, K. (1990). Foundations of distance education. London: Routledge

Horvat, M., (2013, July 1-4). CESAER, 30th Anniversary of the EU RTD Framework Programme: Achievements and lessons learned, 19th Annual EARMA Conference, Vienna, University of Technology, Five-Year Assessment Report of the EC RTD Framework Programme, Report of the Independent Expert Pannel.

Simeonova-Ganeva, R. And Ganev, K., (2013). Effect of EU Funded Policies: Net Impact Assessment of Investments in Education and Science in Bulgaria.

Bendis, R. and Byler, E. (2009). Creating a national innovation framework.

Jongsma, A. (2002). TEMPUS @ 10 A Decade of University Cooperation, DG Education and culture. 